\title{
Imaging Molecular Structure and Charge Simultaneously
}

\author{
A new technique determines both the charge distribution and the \\ structural distortions that result from the addition of a single charge to a \\ molecule.
}

By Mark Buchanan

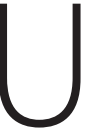
sing molecules called conducting polymers as tiny, current-carrying wires, researchers hope to assemble devices from atomic-scale components. Now a team has developed a technique that addresses theoretical uncertainties about the responses of these molecules to extra charges [1]. The team combined a technique for imaging charge with one for imaging structure and found that for a short polymer strand, an added charge remains confined in a small region, rather than spreading out. They also learned that the charge only distorts a small segment of the molecular structure.

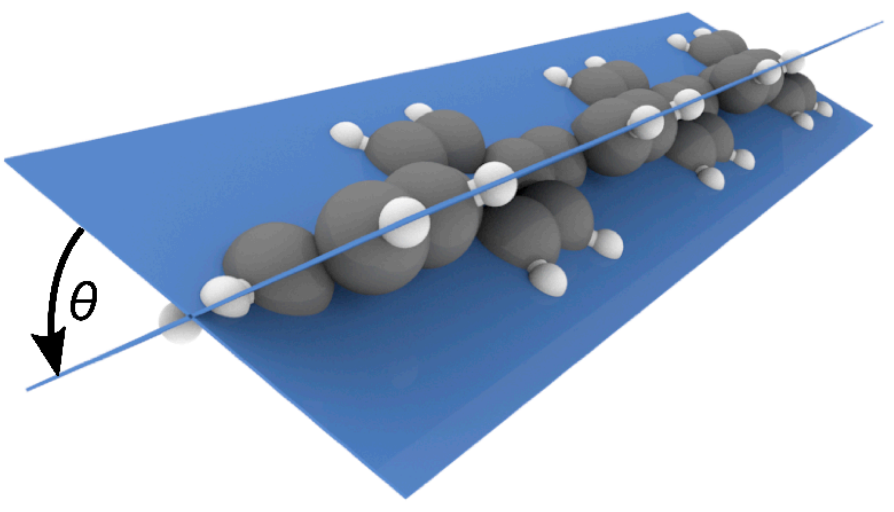

At full tilt. This polymer chain is made from six phenylene units, hexagons of six carbon atoms (gray) with four hydrogen atoms attached (white). In the uncharged state, the planes of neighboring rings are tilted with respect to each other at an angle $(\theta)$ of about $34^{\circ}$. The addition of a single charge leads to a reduction in this angle for the rings near the polymer's center.

Credit: L. L. Patera et al. [1]
The atomic-scale information that the technique provides will help researchers better understand the process by which these polymers carry current.

Adding a single charge to a conducting polymer can produce a distortion of the molecule's structure. The combination of the charge with the associated distortion is known as a polaron, a quasiparticle that can hop along the polymer chain as part of a current. But the detailed properties of polarons, which determine the electronic behavior of the polymer, have remained difficult to determine experimentally. In addition, various theoretical predictions have not agreed on matters such as the amount of structural distortion or the degree to which the charge spreads out.

To answer these questions with an atomic-scale probe-and provide a test bed to improve theoretical models-Jascha Repp and colleagues, from the University of Regensburg in Germany, have now combined two techniques. The first is a standard method to determine molecular structure using an atomic force microscope (AFM). The second is a system for mapping out the charge distribution in a molecule by applying an ac voltage to the probe tip of a scanning tunneling microscope (STM). Last year, Repp and his colleagues demonstrated this second technique, which they call AC-STM [2].

The team studied a short polymer consisting of six units of phenylene-a six-carbon-atom ring-resting on a solid surface. First, they used the AFM to find the molecular structure in the absence of any excess charge. As expected from previous studies, this baseline experiment revealed that neighboring rings were tilted by roughly $34^{\circ}$ with respect to one another, 


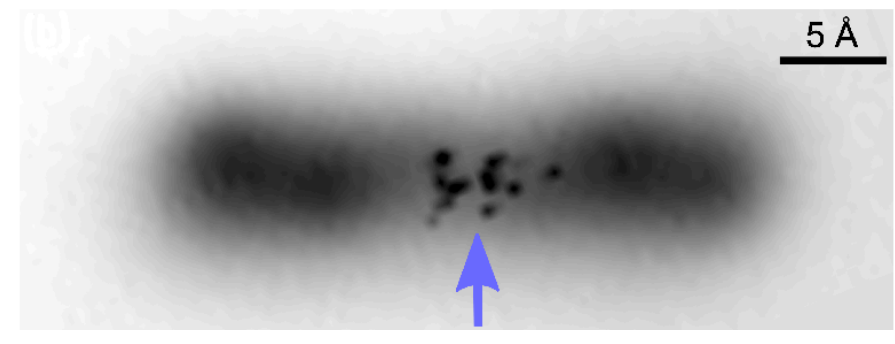

Where's the charge? This AC-STM image of the polymer molecule on which a positive charge has been deposited shows black dots indicating electron tunneling events, where an electron jumps from the microscope tip onto the molecule and back. These events are more likely in the proximity of the positive charge, which is clearly localized in the central region.

Credit: L. L. Patera et al. [1]

rather than residing in a common plane.

Next, Repp and colleagues added a single positive charge to the polymer and measured it using both the AFM and the AC-STM techniques. These measurements revealed that the added charge confined itself to a small region in the center of the polymer and that near the center, the tilt angle between adjacent units had been reduced. So the polymer was flatter in this central region than at the ends of the molecule, where the original structure remained.
Repp says that his team's work marks the first time that experimenters have controlled the charge state of a prototypical conducting polymer while simultaneously imaging its structure and charge distribution. "Theoretical modeling has failed to predict the spatial extent" of excess charge in a polymer, says Regensburg team member Laerte Patera. But the new results "will provide a benchmark for further theoretical investigation," he says.

"Polarons form in essentially all materials and influence their physical and chemical properties," says surface physicist Martin Setvin of the Technical University of Vienna. "But for the last 70 years researchers could only probe them indirectly." He says that the demonstration of a direct probe will be helpful for future understanding of polarons.

Mark Buchanan is a freelance science writer who splits his time between Abergavenny, UK, and Notre Dame de Courson, France.

\section{REFERENCES}

1. L. L. Patera et al., "Imaging charge localization in a conjugated oligophenylene," Phys. Rev. Lett. 125, 176803 (2020).

2. L. L. Patera et al., "Mapping orbital changes upon electron transfer with tunnelling microscopy on insulators," Nature 566, 245 (2019). 